\title{
Interpenetrating multiwall carbon nanotube electrodes for organic solar cells
}

\author{
Anthony J. Miller, ${ }^{\text {a) }}$ Ross A. Hatton, and S. Ravi P. Silva \\ Nano-Electronics Centre, Advanced Technology Institute, University of Surrey, Guildford, \\ Surrey GU2 7XH, United Kingdom
}

(Received 3 April 2006; accepted 5 August 2006; published online 27 September 2006)

\begin{abstract}
Low concentrations of multiwall carbon nanotubes (MWCNTs) $(\sim 1$ wt $\%)$ uniformly distributed within the donor layer of a heterojunction organic solar cell are shown to be an effective means of greatly reducing cell series resistance without compromising cell shunt resistance, resulting in an increase in cell fill factor of 50-60\%. By chemically functionalizing MWCNTs with polar surface moieties, it is also possible to manipulate device open circuit voltage. The results of this study demonstrate that MWCNTs have excellent potential as a versatile interpenetrating electrode material for organic solar cells. (C) 2006 American Institute of Physics. [DOI: 10.1063/1.2357844]
\end{abstract}

Organic solar cells have considerable potential as an economically viable path to harvesting energy from sunlight. ${ }^{1}$ The primary obstacles to realizing applications are the poor operational lifetime and low power conversion efficiency of these devices. ${ }^{1}$ The former principally results from the susceptibility of conjugated polymers to photodegradation, ${ }^{2}$ thermal degradation, ${ }^{2}$ and detrimental doping by atmospheric oxygen. ${ }^{3}$ However, advances in polymer stability and encapsulation techniques promise to overcome lifetime issues for short to medium lifetime applications. ${ }^{4}$ In order to maximize the power conversion efficiency in these devices the short circuit current density $\left(J_{\mathrm{sc}}\right)$, open circuit voltage $\left(V_{\mathrm{oc}}\right)$, and fill factor $(\mathrm{FF})$ must all be maximized. A number of researchers have investigated the possibility of utilizing single wall carbon nanotubes (SWCNTs) as the electron acceptor and electron extracting electrode in heterojunction organic solar cells. ${ }^{5,6}$ Unfortunately, while these devices exhibit large $V_{\text {oc }}$, the $J_{\text {sc }}$ remains low as compared to cells employing Buckminsterfullerene $\left(\mathrm{C}_{60}\right)$ as the acceptor material. Semitransparent SWCNT films have also been demonstrated to be potentially low cost alternatives to the indium tin oxide (ITO) glass electrode in bulk heterojunction organic solar cells. ${ }^{7-9}$ This letter reports the results of investigations aimed at utilizing multiwall carbon nanotubes (MWCNTs) as an interpenetrating holeextracting electrode in bilayer organic solar cells based on the model heterojunction, poly $(m$-phenylenevinylene-co-2,5dioctyloxy- $p$-phenylenevinylene) $(\mathrm{PmPV}) / \mathrm{C}_{60}$. PmPV was chosen as the model donor material because it absorbs strongly in the visible spectrum and is known to solubilize MWCNTs without the need for covalent functionalization. ${ }^{10}$ Unlike SWCNTs - which are invariably a mixture of metallic and semiconducting nanotubes-all MWCNTs exhibit metallic conductivity, making them a more reliable prospect as an electrode material. This study demonstrates the utility of MWCNTs as a versatile, interpenetrating, hole-extracting electrode material for organic solar cells.

High purity $(>90 \%)$ pristine and hydroxyl functionalized MWCNTs grown by chemical vapor deposition were obtained commercially (Nanocyl) and used as received. The typical diameter and length of the MWCNTs were confirmed

${ }^{a)}$ Electronic mail: anthony.miller@surrey.ac.uk by transmission electron microscopy to be $10 \mathrm{~nm}$ and several microns, respectively.

Fluorinated MWCNTs were prepared by reaction of pentadecafluoro-octanoyl chloride with hydroxyl functionalized MWCNTs. The coupling reaction was performed under nitrogen at $90^{\circ} \mathrm{C}$ for a period of $20 \mathrm{~h}$. The functionalized tubes were repeatedly washed with chloroform over a $0.2 \mu \mathrm{m}$ polytetrafluoroethylene filter to remove unreacted acid chloride and subsequently dried prior to the device fabrication. Stable dispersions of pristine and functionalized MWCNTs in chloroform solutions of PmPV (Sigma-Aldrich) were prepared by ultrasonically dispersing carbon nanotubes in chloroform prior to adding PmPV. Devices were fabricated on ITO coated glass precoated with $50 \mathrm{~nm}$ poly (3,4-ethylenedioxythiophene): poly (styrenesulfonate) (PEDOT:PSS). PmPV solutions containing 0\%, 0.25\%, $0.5 \%, 1 \%$, and $2 \%$ MWCNTs by weight (wt \%) were spin coated onto the PEDOT:PSS coated ITO glass substrates to a thickness of $50 \mathrm{~nm}$. $\mathrm{C}_{60}(99.9 \%)$ was deposited under high vacuum at $0.2 \mathrm{~nm} \mathrm{~s}^{-1}$ to a thickness of $50 \mathrm{~nm}$ followed by deposition of a $50 \mathrm{~nm}$ aluminum counter electrode. The photoactive device area was typically $5 \mathrm{~mm}^{2}$.

Owing to their high aspect ratio, MWCNTs generally tend to aggregate in solution and have an extremely low percolation threshold in the solid state. ${ }^{11} \mathrm{PmPV}$ forms stable dispersions of MWCNTs in chloroform, because the conjugated backbone wraps around the graphitic tubes, rendering them soluble and inhibiting the attractive van der Waals interactions between them. ${ }^{10}$ For thin film optoelectronic applications it is imperative that the MWCNTs are uniformly distributed within thin films cast from solution. Scanning electron microscopy (SEM) studies reveal that for MWCNT loadings $\leqslant 2$ wt $\%$ nanotubes are uniformly distributed within the PmPV layer, when spin cast onto PEDOT:PSS coated ITO glass (Fig. 1). Furthermore, the peak-to-peak roughness of the said films, measured using an atomic force microscope, is less than $10 \mathrm{~nm}$, confirming that for MWCNT loadings $\leqslant 2$ wt $\%$ carbon nanotubes do not protrude from the film surface, but are confined within the nominal film thickness of $50 \mathrm{~nm}$. Consequently, the focus of this investigation is on MWCNT loadings $\leqslant 2 \mathrm{wt} \%$.

According to the metal-insulator-metal (MIM) model, the built-in potential across a thin film of an intrinsic, wide band gap material is equal to the difference in work function 


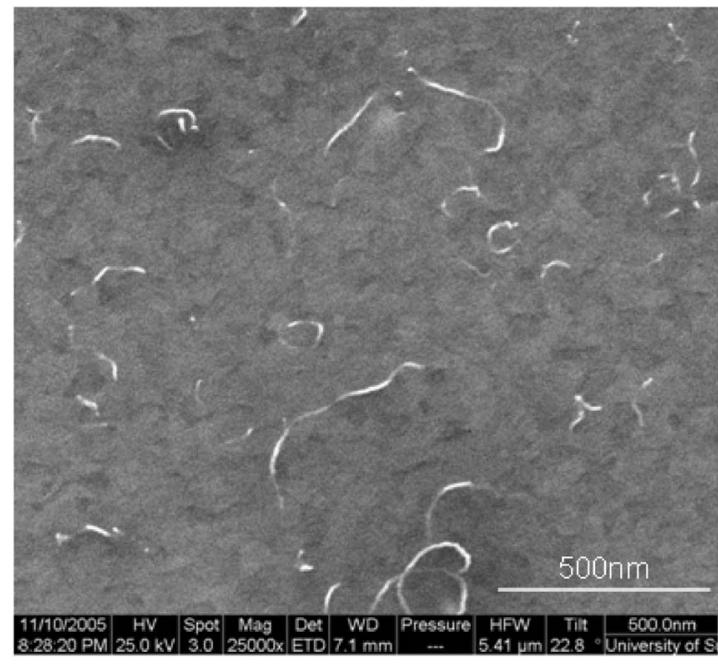

FIG. 1. Representative SEM image of a $50 \mathrm{~nm}$ film of PmPV/1 wt \% MWCNTs spin cast onto PEDOT:PSS coated ITO glass. The polycrystalline structure of the underlying ITO is clearly visible, indicating that the PmPV and PEDOT:PSS layers are sufficiently thin to be semitransparent to secondary electron emission.

between the contact electrodes. ${ }^{12}$ Figure 2 shows how $V_{\mathrm{oc}}$ varies as a function of MWCNT loading within the PmPV layer. The $V_{\mathrm{oc}}$ of cells without carbon nanotubes, $0.75 \mathrm{~V}$, is consistent with the difference in work function between the $\mathrm{Al}$ cathode $(4.25 \mathrm{eV})$ and PEDOT:PSS anode $(5.0-5.1 \mathrm{eV})$.

For MWCNT loadings $>0.25 \mathrm{wt} \%, V_{\mathrm{oc}}$ is reduced by $\sim 0.35 \mathrm{~V}$ to a constant value of $\sim 0.4 \mathrm{~V}$. Literature values for the work function of MWCNTs vary between 4.3 and $4.95 \mathrm{eV} .{ }^{13-15}$ This spread of values reflects the sensitivity of work function to surface defects and contamination, which depend strongly on the method of carbon nanotube synthesis and purification. According to the MIM model, the work function of the MWCNTs used here is $\sim 4.65 \mathrm{eV}$, which falls within the range of reported values determined directly using photoelectron spectroscopy. The reduction in $V_{\text {oc }}$ to a constant value of $\sim 0.4 \mathrm{~V}$ indicates that the MWCNTs are in electrical contact with the PEDOT:PSS coated ITO electrode rather than isolated within the PmPV layer. Evidence that the reduction in the built-in potential cannot be attributed to partial shorting of the electrodes by the carbon nanotubes is twofold (i) $V_{\text {oc }}$ converges to $\sim 0.4 \mathrm{~V}$ with increasing carbon nanotube concentration, rather than continuing to decline, and (ii) the cell shunt resistance (indicated by the gradient of the fourth quadrant response at $0 \mathrm{~V}$ ) remains unchanged when multiwall carbon nanotubes are introduced into the PmPV layer, indicating that the carbon nanotubes do not enhance current leakage across the device at the loading and dispersion we have used in our cells.

The cell FF is increased when MWCNTs are introduced into the PmPV layer, owing to a sharp rise in forward current. This change in the current-voltage characteristics is consistent with a reduction in cell series resistance. For carbon nanotube loadings $\geqslant 1 \mathrm{wt} \%$, the FF is increased by a factor of 1.6-1.7, as compared to the reference device. This increase in FF is a function of carbon nanotube loading and appears to saturate for loadings $\geqslant 1 \mathrm{wt} \%$. The cell series resistance is simply the sum of contributions from bulk transport within the organic layers, carrier extraction at the electrodes, and transport through the contacts to the external circuit. The current-voltage characteristics of single layer diodes comprising a $50 \mathrm{~nm}$ layer of PmPV with and without Downloaded 30 Mar 2009 to 131.227.178.132. Redistribution subject to AIP license or copyright; see http://apl.aip.org/apl/copyright.jsp

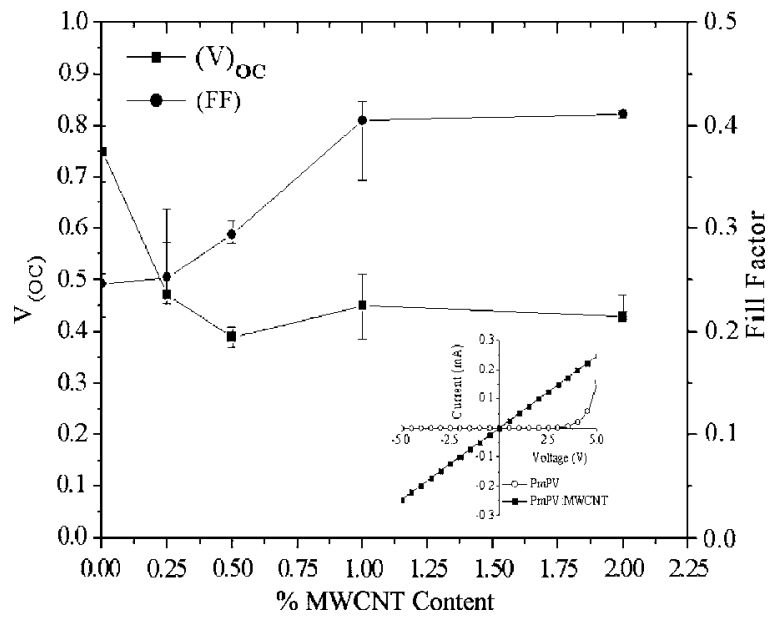

FIG. 2. Summary of $V_{\mathrm{oc}}$ and FF of the bilayer heterojunction organic solar cell, ITO/PEDOT:PSS/PmPV/C $60 / \mathrm{Al}$, as a function of wt $\%$ MWCNTs within the PmPV layer, under 1 sun $\left(100 \mathrm{~mW} \mathrm{~cm}^{-2}\right)$ simulated solar illumination. The lines connecting the data points are included to guide the eye and have no physical meaning. Inset: $I-V$ characteristics of single layer

MWCNTs [Fig. 2 (inset)] provide direct evidence for the percolation of MWCNTs throughout the PmPV layer, since devices incorporating MWCNTs are Ohmic. The reduction in series resistance may therefore be attributed to a reduction in the "average" distance over which positive charge carriers (holes) formed at the organic heterojunction must traverse before extraction by the anode. While it is unlikely that all of the holes formed at the organic heterojunction are extracted by MWCNTs, we suggest that geometrical field enhancement at the carbon nanotubes greatly increases the range over which positive charge carriers can be efficiently extracted by each MWCNT. The electric field enhancement factor in this configuration is estimated to be as great as five fold. ${ }^{16,17}$

In thin film $(<100 \mathrm{~nm})$ organic solar cells, charge carriers formed at the organic heterojunction are extracted by the built-in electric field and to a lesser extent the carrier concentration gradients. Consequently, a change in $J_{\mathrm{sc}}$ is indicative of a change in the electric field strength across the organic layers. ${ }^{18}$ When MWCNTs are incorporated into the PmPV layer, $J_{\mathrm{sc}}$ is reduced from $1.0 \mathrm{~mA} \mathrm{~cm}^{-2}$ in the reference cell to $0.8 \mathrm{~mA} \mathrm{~cm}^{-2}$ for MWCNT loadings of $0.5-2$ wt \% within the PmPV layer.

Arguably the effect on the electric field strength of the $50 \%$ reduction in the built-in potential in cells utilizing MWCNTs is almost offset by the effective reduction in the electrode separation, since the MWCNTs extend throughout the PmPV layer. However, Fig. 1 clearly shows that the density of MWCNTs within the PmPV layer is very low. Consequently, the mean geometric reduction in the electrode separation is very small. Such a small reduction in $J_{\mathrm{sc}}$ is evidence that the MWCNTs efficiently extract positive charge carriers formed at the organic heterojunction. It is energetically favorable for holes to be transferred from the highest occupied molecular orbital of PmPV (5.8 eV) (Ref. $19)$ to the Fermi level of MWCNTs $(\sim 4.6 \mathrm{eV})$, whereupon they are extracted to the external circuit along the highly conductive MWCNTs. We suggest that geometrical field enhancement at the sidewalls and tips of the MWCNTs also contributes to efficient positive charge carrier extraction.

Postfabrication annealing of bilayer organic solar cells can dramatically increase $J_{\mathrm{sc}}{ }^{20}$ This occurs because the moldiodes comprised of PmPV and PmPV with 1 wt \% MWCNTs. 


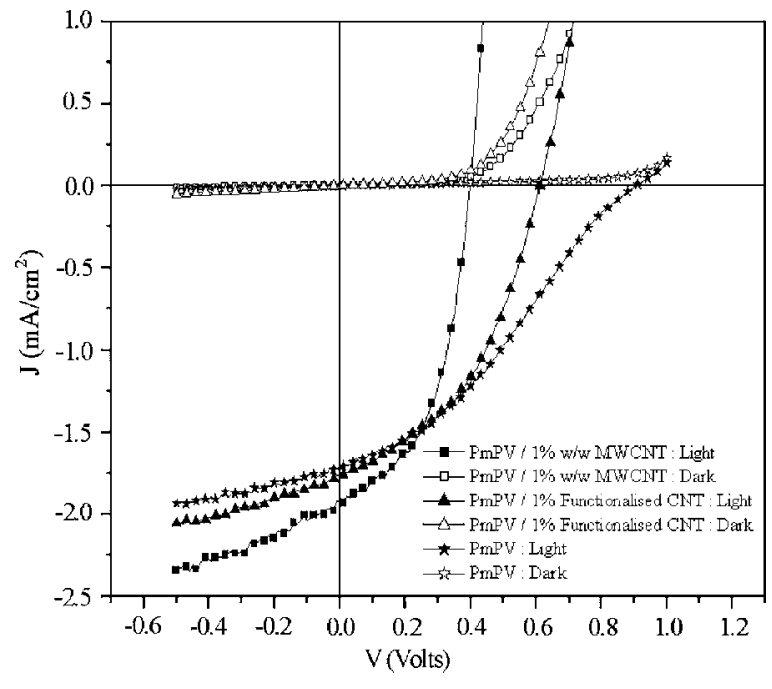

FIG. 3. Typical current-voltage characteristics in the dark (empty shapes) and under 1 sun simulated solar illumination (filled shapes) of the annealed reference, MWCNT, and functionalized MWCNT devices.

ecules comprising each layer are sufficiently thermally agitated to interdiffuse, thereby increasing the heterojunction area. We have investigated the effect of annealing cells with and without $1 \mathrm{wt} \%$ MWCNTs within the donor layer. Complete cells were heated in an inert atmosphere to a peak temperature of $120^{\circ} \mathrm{C}$ for $5 \mathrm{~min}$. Typical current-voltage characteristics made in the dark and with 1 sun simulated solar illumination are shown in Fig. 3.

For cells with and without MWCNTs, the cell fill factors are increased to 0.48 and 0.32 , respectively. The fill factor of cells incorporating $1 \mathrm{wt} \%$ MWCNTs within the PmPV layer remains $50 \%$ larger than that of the reference. $V_{\mathrm{oc}}$ in the reference cell is increased by $0.15 \mathrm{~V}$ upon annealing, while that of the MWCNT device remains unchanged at $\sim 0.4 \mathrm{~V}$. In cells with and without MWCNTs, $J_{\mathrm{sc}}$ is significantly increased. Notably cells employing MWCNTs within the $\mathrm{PmPV}$ layer exhibit the greatest increase in $J_{\mathrm{sc}}$ and largest $J_{\mathrm{sc}}$. This result is further evidence that the MWCNTs efficiently extract photogenerated positive charge carriers from within the device.

The power conversion efficiency of annealed cells utilizing MWCNTs within the PmPV layer remains lower than that of the reference $(0.37 \%$ and $0.5 \%$, respectively). This is because of the very large $V_{\mathrm{oc}}$ in the reference devices. In order to translate the large enhancement in cell fill factor and increase in $J_{\mathrm{sc}}$ achieved by incorporating MWCNTs within the PmPV layer into an improvement in power conversion efficiency, $V_{\text {oc }}$ must be increased. According to the MIM model increasing the MWCNT work function should result in a commensurate increase in $V_{\text {oc }}$. To test this hypothesis the outer surface of pristine MWCNTs was acid oxidized, a treatment which is known to increase the work function by as much as $0.8 \mathrm{eV} .{ }^{15}$ Unfortunately oxidized MWCNTs could not be dispersed in PmPV, highlighting the need for compatibility between the surface functionality and the conjugated polymer. To facilitate compatibility with PmPV while increasing the work function, MWCNTs were derivatized, via ester linkages, with the electron withdrawing functionality, pentadecafluoro-octanoyl chloride. Previous studies have shown that monolayers of fluorinated alkanes covalently bonded to electrode surfaces increase the substrate work function by increasing the surface potential. ${ }^{21}$ MWCNTs functionalized in this way readily disperse in chloroform solutions of PmPV. Furthermore, when incorporated into annealed cells a $0.21 \mathrm{~V}$ increase in $V_{\text {oc }}$ is achieved, consistent with an increase in the MWCNT work function as compared to pristine MWCNTs (Fig. 3). This result is further evidence that MWCNTs function as an electrode in this context. Crucially, this increase in $V_{\text {oc }}$ is achieved while retaining a large FF (0.43), demonstrating the viability of this approach. In this particular case the increase in $V_{\text {oc }}$ raises the power conversion efficiency to $0.46 \%$ which is comparable to that of the reference. However, efforts to identifying suitable functionality to achieve a further increase in $V_{\mathrm{oc}}$ are currently under way, which, based on the results of this study, is expected to translate the large increase in FF into a significant increase in power conversion efficiency.

The results of this study have demonstrated that MWCNTs have strong potential as an interpenetrating, holeextracting electrode in organic solar cells. Chemically functionalizing the outer surface of MWCNTs with polar moieties is shown to be an effective means of tuning $V_{\text {oc }}$ and the key to realizing the ultimate goal of improved power conversion efficiency using this approach. Crucially, interpenetrating MWCNT electrodes can be incorporated into the device structure without complicating the process of device fabrication.

The authors would like to thank the EPSRC for funding via the Portfolio Partnership scheme.

${ }^{1}$ S. E. Shaheen, D. S. Ginley, and G. E. Jabbour, Mater. Res. Bull. 30, 10 (2005).

${ }^{2}$ L. J. Rothberg, M. Yan, F. Papadimitrakopoulos, M. E. Galvin, E. W. Kwock, and T. M. Miller, Synth. Met. 80, 1 (1996).

${ }^{3}$ S. Heutz, P. Sullivan, B. M. Sanderson, S. M. Schultes, and T. S. Jones, Sol. Energy Mater. Sol. Cells 83, 229 (2004).

${ }^{4}$ C. J. Brabec, J. A. Hauch, P. Schilinsky, and C. Waldauf, Mater. Res. Bull. 30, 50 (2005).

${ }^{5}$ E. Kymakis, I. Alexandrou, and G. A. J. Amaratunga, J. Appl. Phys. 93, 1764 (2003).

${ }^{6}$ B. J. Landi, R. P. Raffaelle, S. L. Castro, and S. G. Bailey, Prog. Photovoltaics 13, 165 (2005).

${ }^{7}$ A. du Pasquier, H. E. Unalan, A. Kaneal, S. Miller, and M. Chhowalla, Appl. Phys. Lett. 87, 203511 (2005).

${ }^{8}$ J. van de Lagematt, T. M. Barnes, G. Rumbles, S. E. Shaheen, T. J. Coutts, C. Weeks, I. Levitsky, J. Peltols, and P. Glatkowski, Appl. Phys. Lett. 88, 233503 (2006).

${ }^{9}$ M. W. Rowell, M. A. Topinka, M. D. McGehee, H. Prall, G. Dennler, N. S. Sariciftci, L. Hu, and G. Gruner, Appl. Phys. Lett. 88, 233506 (2006).

${ }^{10}$ S. A. Curran, P. M. Ajayan, W. J. Blau, D. L. Carroll, J. N. Coleman, A. B. Dalton, A. P. Davey, A. Drury, B. McCarthy, S. Maier, and A. Strevens, Adv. Mater. (Weinheim, Ger.) 10, 1091 (1998).

${ }^{11}$ B. Kilbride, J. Coleman, J. Fraysse, P. Fournet, M. Cadek, A. Drury, S. Hutzler, S. Roth, and W. Blau, J. Appl. Phys. 92, 4024 (2002).

${ }^{12}$ S. M. Sze, Physics of Semiconductor Devices (Wiley, New York, 1981), p. 553.

${ }^{13}$ M. Shiraishi and M. Ata, Carbon 39, 1913 (2001).

${ }^{14}$ O. Gröning, O. M. Küttel, C. Emmenegger, P. Gröning, and L. J. Schlapbach, Vac. Sci. Technol. 18, 665 (2000).

${ }^{15}$ H. Ago, T. Kugler, F. Cacialli, W. Salaneck, M. Shaffer, A. H. Windle, and R. H. J. Friend, J. Phys. Chem. B 103, 8116 (1999).

${ }^{16}$ H. C. J. Miller, J. Appl. Phys. 38, 4501 (1967).

${ }^{17}$ C. J. Edgecombe and U. Valdre, J. Microsc. 203, 188 (2000).

${ }^{18}$ V. D. Mihailetchi, L. J. A. Koster, and P. W. M. Blom, Appl. Phys. Lett. 85, 970 (2004).

${ }^{19}$ D. O'Brien, A. Bleyer, D. G. Lidzey, and D. D. C. Bradley, J. Appl. Phys. 82, 2662 (1997)

${ }^{20}$ M. Drees, R. M. Davies, and J. R. Heflin, Phys. Rev. B 69, 165320 (2004).

${ }^{21}$ B. Choi, J. Rhee, and H. H. Lee, Appl. Phys. Lett. 79, 2109 (2001). 